\title{
NEUIGKEITEN ZU AUSBILDUNG UND FORTBILDUNG!
}

\section{Liebe Kolleginnen und Kollegen!}

Das Ausbildungs-Curriculum für den Fähigkeitsausweis Akupunktur/TCM besteht im Wesentlichen aus vier Pflichtmodulen. Das ModulTCM-Grundlagen findet jährlich an jeweils 26 Kursabenden mit drei Lektionen am Donnerstag in Zürich statt. Das bedeutete bisher für viele Kollegen einen nicht unerheblichen Zeitaufwand, was speziell für Niedergelassene organisatorisch schwierig $\mathrm{zu}$ bewältigen war. Nicht jeder lebt in Zürich und kann mit einer kurzen Tramfahrt in denVorlesungssaal kommen. Aus dem Grund wird dieses Modul ab diesem Jahr nun durchgehend digital angeboten. Wir konnten im letzten Zyklus gute Erfahrungen mit dem Modus «Flipped Classroom» machen. Dabei werden wir den Inhalt des Kursabends die zwei Wochen zuvor als Stream anbieten. Dies ermöglicht den Teilnehmern, sich den Stoff angenehm zu Hause, zur Zeit ihrer Wahl, anzueignen. Anschließend steht am Kursabend der jeweilige Referent für eine interaktive Lektion zurVerfügung. Dieses Format wurde bereits beim vorgängigen Kurszyklus getestet und von vielen Teilnehmern als bereichernd empfunden. Geschätzt wurden einerseits die zeitliche Flexibilität und andererseits auch der fehlende Reiseaufwand, der damit ganz wegfällt.

Ich möchte jetzt schon auf zwei weitere Neuerungen in unserem Kurswesen hinweisen. Speziell im Bereich Fortbildung sollte das Angebot der SACAM erweitert werden. Im Sinne einer Vorabinformation kann ich Ihnen bereits folgendes verraten:
Die „Fachtagungs-Reihe TCM“ ist im Moment noch im Aufbau. Das Format beerbt den früheren Modul-Zyklus ",Akupunktur II“, geht jedoch inhaltlich über die Akupunktur hinaus. Wie wir das schon im Aufbau-Modul «RAC 2/Aurikulomedizin» handhaben, geht der Zyklus mit insgesamt 80 Kursstunden über zwei Jahre. Es können zudem auch einzelne Kurstage gebucht werden. Das Programm wird Ihnen bald kommuniziert werden.

Dem Wunsch einer niedrigschwelligen Fortbildung möchten wir mit einem monatlichen Kursabend nachkommen.Jeden ersten Mittwochabend des Monats planen wir zwei Lektionen per Video zu den verschiedensten Themen. So können Patientenfälle präsentiert werden und Vorträge abgehalten werden. Geplant sind auch Angebote, in denen eigene komplexe Therapie-Fälle besprochen werden können sowie ein Q\&A, Questions \& Answers zu verschiedenen Themen im Bereich der Aurikulomedizin. Es versteht sich von selbst, dass Fortbildung-Credits für die Rezertifizierung des Fähigkeitsausweises vergeben werden. Auch hierzu werden Sie in Kürze zu den geplanten Themen informiert werden. Die Kursleitung der beiden letztgenannten Fortbildungsformate hat Erika Süess.

Ihr

C. Scholtes

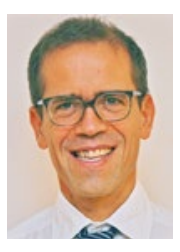

Dr. med. Christoph Scholtes

Co-Präsident der Schweizerischen Ärztegesellschaft für Akupunktur - Chinesische Medizin - Aurikulomedizin, SACAM

Wattenwylweg 21, 3006 Bern

Tel. +41(0)844200200

info@sacam.ch, www.sacam.ch 